\title{
Experiencia del cliente, confianza y lealtad de los millennials en el sector bancario de la ciudad de Cuenca-Ecuador
}

\section{Customer experience, trust and loyalty of millennials in banking at Cuenca-Ecuador}

\begin{abstract}
Mg. Adriana Priscila Vallejo-Bojorque es profesora e investigadora de la Universidad Politécnica Salesiana-UPS (Ecuador) y candidata a Doctora en la Universidad Popular Autónoma del Estado de Puebla-UPAEP (México) (adrianapriscila.vallejo@upaep. edu.mx) (https://orcid.org/0000-0001-8725-836X)
\end{abstract}

Dra. Judith Cavazos-Arroyo es profesora e investigadora en el decanato de Ciencias Económico-Administrativas de la Universidad Popular Autónoma del Estado de Puebla-UPAEP (México) (judith.cavazos@upaep.mx) (https://orcid.org/0000-0002-6258-289X)

Dr. Mario Alberto Lagunez-Pérez es profesor e investigador en el decanato de Ciencias Económico-Administrativas de la Universidad Popular Autónoma del Estado de Puebla- UPAEP (México) (marioalberto.lagunes@upaep.mx) (https://orcid.org/0000-0002-1548-5114)

Dra. Sofía Elba Vásquez-Herrera es profesora e investigadora en el decanato de Ciencias Económico-Administrativas de la Universidad Popular Autónoma del Estado de Puebla-UPAEP (México) (sofiaelba.vazquez@upaep.mx) (https:// orcid.org/0000-0001-8053-5678)

\section{Resumen}

El objetivo de esta investigación fue explicar el efecto de la experiencia del cliente bancario sobre la confianza y el efecto de esta última sobre la lealtad del cliente, logrando así complementar investigaciones anteriores donde se sugiere explorar con claridad la red nomológica de los constructos que actúan como mediadores en la relación entre la experiencia del cliente y la fidelidad del mismo. La muestra estuvo conformada por 1231 usuarios de la banca de la ciudad de Cuenca-Ecuador que forman parte de la generación conocida como millennials, segmento de interés por sus características y comportamiento de compra. Para el análisis de los datos se utilizó el modelo de ecuaciones estructurales y el software PLS (Partial Least Square). Los resultados evidencian que existe un efecto significativo de la experiencia del cliente sobre la confianza en la banca y a su vez un efecto similar entre la confianza y lealtad del cliente; además, se confirma que la confianza representa una variable que antecede a la lealtad. Los rasgos y comportamientos de los millennials, conocedores de la tecnología e Internet, evidencian una relación entre las variables que son parte del modelo propuesto y aplicado en el sector bancario.

\begin{abstract}
This paper aims to explain the effect of the banking customer experience on trust and the effect of the latter on customer loyalty, thus complementing previous research where it is suggested to clearly explore the nomological network of the constructs that act as mediators in the relationship between customer experience and customer loyalty. The sample consisted of 1231 users of the bank in Cuenca-Ecuador who are part of the generation known as millennials, a segment of interest due to their great attachment to technology and purchasing behavior. The structural equations model and PLS (Partial Least Square) software were used for data analysis. The results show that there is a significant effect of customer experience on trust in banking and in turn a similar effect between customer trust and loyalty. Furthermore, it is confirmed that trust represents a variable that precedes loyalty. The behaviors of millennials as they are knowledgeable about technology and experts in using the Internet to search and purchase products, show a relationship between the variables that are part of the proposed model that is applied in the banking sector.
\end{abstract}

\section{Palabras clave I keywords}

Banca digital, confianza, experiencia del cliente, lealtad.

Digital banking, trust, customer experience, loyalty.

Cómo citar: Vallejo-Bojorque, A.P., Cavazos-Arroyo, J., Lagunez-Pérez, M.A., y Vásquez-Herrera, S.E. (2021). Experiencia del cliente, confianza y lealtad de los millennials en el sector bancario de la ciudad de Cuenca-Ecuador. Retos Revista de Ciencias de la Administración y Economía, 11(22), pp. 287-300. https://doi.org/10.17163/ret.n22.2021.06 


\section{Introducción}

La aparición de internet y su influencia en la sociedad trajo consigo la modificación en el comportamiento del consumidor, muchas de las actividades que en décadas pasadas se realizaban únicamente de forma presencial, hoy en día se han complementado con productos y servicios ofrecidos de forma virtual. El sector de la banca sin duda, ha sido parte de los adoptadores tempranos de tecnología computacional, por lo tanto, de una acelerada evolución en el formato digital (Agarwal, 2020). En este contexto, existen varios investigadores que coinciden en que la percepción de los consumidores, con respecto al uso de la banca en línea, involucra altos niveles de riesgo, ya que los servicios vinculados a una tecnología emiten estímulos poco familiares y como consecuencia, los consumidores que deciden usar los servicios bancarios online, se ven inmersos en una situación de incertidumbre afectando así los niveles de confianza (Sarin et al., 2003).

Con el objetivo de captar mayor cantidad de clientes y sobre todo de fidelizar a los actuales, varias instituciones optaron por el desarrollo tecnológico; este avance en el sector bancario y de la economía, hoy son muy conocidos y agregan valor al servicio por el vínculo que generan con los usuarios, rebasando la antigua banca de solo productos para concentrarse en una nueva banca, dinámica y de enfoque al cliente (Mahecha et al., 2019). Autores como Pikkarainen et al. (2006) indican que en sí, la banca electrónica ha evidenciado un crecimiento constante en términos de valor y de volumen.

El sector de los servicios bancarios ha sido muy trabajado a nivel de investigaciones académicas; sin embargo, autores como Kamath et al. (2019) afirman que varias cuestiones siguen sin estar claras, dado que investigaciones anteriores como las de Brakus et al. (2009) y Srivastava y Kaul (2016) han examinado el vínculo entre la experiencia del cliente y la lealtad, confirmando que existe escasez de literatura académica que examine el papel mediador de dichas variables secuenciales, por lo que sugieren explorar con claridad la red nomológica de los constructos que actúan como mediadores en la relación entre la experiencia del cliente y la fidelidad (Kamath et al., 2019). Por ello, esta investigación pretende explicar el efecto de la experiencia del cliente sobre la confianza y el efecto de esta última sobre la lealtad del cliente. Este trabajo, está dividido en las siguientes secciones: revisión de la literatura, metodología, resultados discusión y conclusiones.

\section{Revisión de la literatura}

\subsection{La banca electrónica como avance del sector bancario}

Por varios años la banca puso su servicio a disposición de la comunidad con base en el factor humano, pues era siempre una persona quien brindaba atención a los usuarios. El desarrollo de las áreas de marketing, servicio al cliente y desarrollo de procesos, trajo consigo la introducción de la banca electrónica, este servicio diferenciador, brinda a los clientes la capacidad de realizar transacciones financieras sin la intervención de seres humanos; es decir, posibilita a un usuario acceder a sus cuentas de banco por Internet y realizar una transacción (Safeena et al., 2013). El éxito de las innovaciones de este tipo de servicio, depende en gran medida de los patrones o tendencias de consumo de usuarios de dichos servicios (Arora \& Sandhu, 2018).

La banca electrónica puede ser entendida como el suministro automático de productos y servicios bancarios nuevos y tradicionales directamente a los clientes, por medio de canales interactivos de comunicación electrónica (Drigă \& Isac, 2014), en este 
sentido, el proceso de servicio puede ser definido como la configuración de tecnologías a través de las cuales los proveedores del servicio, perciben y responden a las necesidades dinámicas y complejas de sus clientes utilizando tecnología avanzada (Dabholkar \& Overby, 2005).

Las múltiples innovaciones sobre todo aquellas que se desarrollaron en temas de tecnología, dieron como resultado un aumento significativo en las transacciones de comercio electrónico, así como el desplazamiento del contacto físico a las transacciones electrónicas. El avance del sector bancario y de la economía, hoy son muy conocidos por el vínculo que logra con los usuarios. De acuerdo con Pikkarainen et al. (2006), el sistema de banca electrónica ha evidenciado un crecimiento constante en términos de valor y de volumen.

\subsection{Experiencia del cliente y confianza en el sector bancario}

Los usuarios cada día exigen más en temas de atención y servicio, las organizaciones hacen grandes esfuerzos para proporcionar las facilidades adecuadas para el desarrollo de una experiencia satisfactoria con la marca. En la misma línea, Kavitha y Haritha (2018) definen a la experiencia del cliente como la percepción de los usuarios acerca de cómo los trata una institución, mientras que autores como Buttle (2008) y De-Keyser (2015), coinciden en que la experiencia del cliente involucra elementos cognitivos, emocionales, físicos, sensoriales, espirituales y sociales; es decir, lo entienden como el resultado cognitivo y afectivo de la exposición o interacción del cliente con la gente de una empresa, sus procesos, tecnologías, productos, servicios y demás.

Crear una experiencia de cliente superior, parece ser uno de los objetivos centrales en la mayoría de los entornos actuales, empresas de todo el mundo han adoptado el concepto de gestión de la experiencia del cliente y como resultado muchos incorporan esta particularidad en sus declaraciones de misión. Con respecto a la construcción de la experiencia del cliente, esta es de naturaleza holística e involucra a los clientes, está dada no solo por aquellos elementos que el proveedor puede controlar, sino también por elementos que están fuera del control del proveedor, la experiencia del cliente abarca la experiencia total, incluye la búsqueda, fases de compra, consumo y posventa (Verhoef et al., 2009).

Investigadores como Ali et al. (2018) y Slåtten et al. (2011) consideran que la experiencia del cliente constituye un constructo multidimensional y diverso, desarrollado por varios elementos que incluyen el entorno físico, interacciones con el personal e interacciones con otros clientes. Por su parte, Loureiro et al. (2014) enfatizan en que las emociones de los clientes, principalmente el deleite, son componentes naturales de su experiencia, por lo que una de las claves es provocar fuertes emociones y reacciones experienciales del consumidor, de ahí, que se puede confirmar lo indicado por Slåtten et al. (2011), las experiencias se almacenan en los recuerdos lo que genera un vínculo estrecho entre ellos y su deleite. En este sentido, las experiencias placenteras pueden considerarse un determinante vital de la lealtad del cliente (Ali et al., 2018).

La importancia de comprender las emociones de un usuario en el contexto del servicio que se entregue, proporcionará la posibilidad de crear huellas mentales que contribuyan positivamente con el posicionamiento de una marca (Slåtten et al., 2011). Por lo tanto, un banco debería concentrarse en crear experiencias positivas en los factores que afectan directamente a los comportamientos discrecionales (Wasan, 2018). La parte neural de la gestión de la experiencia del cliente se ha atribuido a intensificar la relación entre empresas y clientes; en el sector de servicios, se ha podido evidenciar aún más por la fuerza de la interacción que exige la banca (Mbama et al., 2018). 
Por otro lado, la confianza ha sido trabajada en varios sectores industriales y definida desde varias perspectivas. Desde los entornos virtuales, Jarvenpaa et al. (2000) definen la confianza en el vendedor online como la expectativa de una de las partes acerca de los motivos y comportamientos de la otra parte. La mayor cantidad de investigaciones realizadas en escenarios electrónicos, consideran a la confianza como un constructo de carácter multidimensional formado por honestidad, benevolencia y competencia, fundamental para conseguir relaciones de compraventa satisfactorias en el contexto mencionado (Doney \& Cannon, 1997; San Martín \& Camarero, 2010).

La honestidad básicamente contempla aquella creencia en que la otra parte asumirá sus compromisos y sus obligaciones, es decir, el cumplimiento de las promesas del socio (Doney \& Cannon, 1997). La benevolencia por su parte, es la creencia en que la otra parte está interesada en conseguir beneficios mutuos y no iniciará temas que perjudiquen la relación (Torres et al., 2009). Finalmente, la competencia constituye la apreciación en la empresa de conocimientos técnicos y experiencia que acrediten el dominio en su campo de actividad, garantiza las condiciones de hacer bien su trabajo y ofrecer un producto o servicio con la calidad ofrecida (Bhattacherjee, 2002; Pavlou, 2003; Roy et al., 2001; Suh \& Han, 2002). Micu et al. (2019) indican que la experiencia del cliente en línea influye directamente en la confianza, por lo que se plantea la siguiente hipótesis:

H1: La experiencia del cliente afecta directa y significativamente a la confianza.

\subsection{Confianza y lealtad en el sector bancario}

La confianza en el contexto de la banca en línea, puede mirarse como aquella creencia que tiene un consumidor en la capacidad del proveedor de servicios de banca por Internet para proporcionar servicios confiables a través de la nube, esto significa que uno está dispuesto a depender de la otra parte a pesar de que no puede controlarla. Con el desarrollo acelerado del mercado virtual, el papel de la confianza se ha vuelto predominante; las incertidumbres potenciales provienen de múltiples fuentes, como la vulnerabilidad de Internet, las plataformas de comunicación de Internet y la capacidad técnica de los proveedores (Bashir \& Madhavaiah, 2015).

La lealtad al servicio es quizá una de las construcciones más importantes en los servicios, de hecho, los clientes leales que se complacen en compras repetidas son la base de cualquier negocio (Shamsuddoha \& Alamgir, 2003). La lealtad del cliente puede ser entendida como el proceso continuo que no termina con la satisfacción de la necesidad del cliente, sino que continúa con la generación de una relación de compra repetida a largo plazo con el cliente en una marca en particular, es decir, la capacidad de una empresa para seguir ganando el patrocinio de un cliente sobre la competencia (Omoregie et al., 2019).

El sector bancario en general ha mostrado una fuerte competencia en el mundo y con el objetivo de garantizar su competitividad en la industria han enfocado sus estrategias en la retención y lealtad de sus clientes (Leninkumar, 2017). El sector de servicios se ha enfrentado a cambios radicales durante los últimos diez años, varias estrategias que se han desarrollado, intentan retener a los clientes. En los servicios bancarios, muchas de las marcas han optado por introducir nuevos productos intangibles y novedosos que son más complejos de imitar (Shamsuddoha \& Alamgir 2003).

La medición de la lealtad del cliente comprende dimensiones como la actitud y el comportamiento. En la misma línea, algunos autores señalan que la satisfacción, la calidad del servicio y la confianza tienen un efecto significativo en la lealtad (Omoregie et al., 2019), también Gillani y Awan (2014) confirman una relación significativa entre la confianza y lealtad del cliente, de ahí que este estudio plantea la siguiente hipótesis: 
H2: La confianza afecta directa y significativamente a la lealtad del cliente.

\section{Materiales y método}

El enfoque de esta investigación fue cuantitativo, el diseño fue de tipo explicativo y la investigación transversal, la técnica a utilizar fue la encuesta, el tipo de muestreo fue no probabilístico y por conveniencia debido a que se determinó un perfil específico de participantes, usuarios (hombres y mujeres) nacidos entre el año 1980 y 1993 , clientes de la banca (Banco del Pacífico, Banco del Austro, Banco Bolivariano, Banco General Rumiñahui, Banco de Guayaquil, Banco Internacional, Banco de Loja, Banco de Machala, Banco del Pichincha, Banco ProCredit, Produbanco, Citibank, Banco Solidario) en la ciudad de Cuenca, la tercera más grande del Ecuador (INEC, 2019).

El levantamiento de información se realizó entre los meses de mayo y julio de 2020 y el estudio se concentró únicamente en personas que son parte de la generación conocida como millennials, pues resulta de interés el comportamiento específico de este grupo, que de acuerdo con Cleyle et al. (2006) integra a quienes tienen un conjunto completamente nuevo de expectativas y requisitos a comparación de las generaciones anteriores, dado que crecieron a la par con la tecnología; es decir, en medio de la era de la información.

La generación millennials es conocida por ciertos rasgos y comportamientos que pueden influir en sus decisiones de compra, a más de ser conocedores de la tecnología, son expertos en usar Internet para la búsqueda y compra de productos, lo utilizan como su principal fuente de información y confían en ella, se preocupan por el medio ambiente y temas de responsabilidad social, buscan y esperan ver publicidad que incluya diversidad de raza y género (Nowak et al., 2006). Su naturaleza en muy optimista, práctica y los caracteriza la creencia de que pueden marcar una diferencia en el mundo (Lancaster \& Stillman, 2002).

La encuesta fue diseñada con base en varios constructos, incluyó 35 ítems. Como parte del constructo "experiencia del cliente" propuesto por Wasan (2018), se consideraron seis dimensiones. Conveniencia, se midió utilizando seis ítems, competencia y compasión por medio de tres ítems cada una y credibilidad, contexto y personalización, con cuatro ítems respectivamente. La lealtad se midió utilizando cinco ítems sugeridos por Moliner et al. (2019) mientras que confianza fue analizada por medio de seis ítems que atienden a lo indicado por Kaur y Arora (2020).

El instrumento incluyó la escala de medición de Likert con un intervalo entre 1 y 5 puntos, fue validado previamente con el Alpha de Cronbach y confirmado con una prueba piloto. La muestra estuvo conformada por 1231 casos y se utilizó el modelo de ecuaciones estructurales y el software PLS (Partial Least Square) para el análisis de los datos.

Este modelo establece la relación de dependencia entre variables, es decir, intenta integrar un conjunto de ecuaciones lineales y establecer cuáles de ellas son dependientes o independientes de otras. Permite examinar todas las variables relevantes simultáneamente y adicional a esto, evaluar el modelo teórico de la investigación y la significancia de las hipótesis. Involucra el modelo de medida, en el que se analizan las cargas factoriales de las variables observables con relación a sus correspondientes variables latentes, en esta estructura se evalúa la fiabilidad y validez de las medidas del modelo teórico (Sáenz \& Tamez, 2014).

También contempla un modelo estructural en el que se analizan las relaciones de causalidad entre las variables latentes independientes y dependientes. Las variables latentes son aquellas que no pueden ser medidas directamente (variables exógenas) y que actúan como variables predictoras de constructos endógenos (Sáenz \&Tamez, 2014). 


\section{Resultados}

\subsection{Evaluación del modelo de medición}

La Tabla 1 proporciona información del perfil demográfico de los encuestados basado en una muestra completa de usuarios de la banca en la ciudad de Cuenca, Ecuador y que son parte de la generación hoy en día conocida como millennials. En la etapa de evaluación del modelo de medición, se realizaron algunos análisis para confirmar la confiabilidad y validez de los datos. En primera instancia, se realizó un análisis factorial confirmatorio (AFC) y se probaron la confiabilidad de las escalas de medición, la validez convergente y la validez discriminante. De acuerdo con los hallazgos, es necesario indicar que la dimensión "competencia" parte del constructo "Experiencia del Cliente" fue eliminada debido a que no cumplió con las cargas mínimas de 0.50 sugerido por Bagozzi et al. (2013).

Tabla 1. Información demográfica de los encuestados

\begin{tabular}{|l|l|r|r|}
\hline \multicolumn{1}{|c|}{ Variable } & \multicolumn{1}{|c|}{ Categoría } & Frecuencia & Porcentaje \\
\hline & Masculino & 598 & $49 \%$ \\
\hline & Femenino & 633 & $51 \%$ \\
\hline Nivel de educación & Educación Básica & 117 & $9.5 \%$ \\
\hline & Bachillerato & 410 & $33.30 \%$ \\
\hline & Tercer Nivel & 600 & $48.74 \%$ \\
\hline & Cuarto Nivel & 104 & $8.45 \%$ \\
\hline Tipo de Vivienda & Suite de lujo & 11 & $0.89 \%$ \\
\hline & Cuarto(s) en casa arrendada & 97 & $7.88 \%$ \\
\hline & Departamento arrendado & 273 & $22.17 \%$ \\
\hline & Casa / Departamento propio & 825 & $67.01 \%$ \\
\hline & Choza/ Covacha /Otro & 25 & $2.03 \%$ \\
\hline Número de vehículos & 0 & 399 & $32.41 \%$ \\
\hline & 1 & 555 & $45.09 \%$ \\
\hline & 2 & 223 & $18.12 \%$ \\
\hline & 3 & 40 & $3.25 \%$ \\
\hline & Más de 3 & 14 & $1.14 \%$ \\
\hline Servicio de Internet & Si & 1165 & $94.64 \%$ \\
\hline & No & 66 & $5.36 \%$ \\
\hline Edad & Promedio & 32.27 & \\
\hline & Edad mínima & 27 & \\
\hline & Edad máxima & 1231 & $100 \%$ \\
\hline & n: & & \\
\hline
\end{tabular}

Los resultados expuestos en la Tabla 2, evidencian la consistencia interna alcanzada dado que todas las cargas de los ítems cumplen con el punto de corte mínimo de 0.50 sugerido por Bagozzi et al. (2013), la confiabilidad compuesta (CR) de todos los constructos, así como la varianza promedio extraída (AVE), fueron mayores a 0.70 y superior 0.50, respectivamente (Chin, 2010; Fornell \& Larcker, 1981) 
Tabla 2. Cargas externas, CR y AVE

\begin{tabular}{|c|c|c|c|c|c|}
\hline Constructo & Dimensiones & Items & $\begin{array}{l}\text { Cargas } \\
\text { Externas }\end{array}$ & $\begin{array}{l}\text { Fiabilidad } \\
\text { compuesta }\end{array}$ & $\begin{array}{c}\text { Varianza } \\
\text { extraída } \\
\text { media (AVE) }\end{array}$ \\
\hline \multirow{20}{*}{$\begin{array}{l}\text { Experiencia } \\
\text { del cliente }\end{array}$} & \multirow{3}{*}{ Compasión } & COMPA1 & 0.757 & \multirow{3}{*}{0.864} & \multirow{3}{*}{0.681} \\
\hline & & COMPA2 & 0.835 & & \\
\hline & & COMPA3 & 0.878 & & \\
\hline & \multirow{4}{*}{ Contexto } & CONT1 & 0.859 & \multirow{4}{*}{0.916} & \multirow{4}{*}{0.731} \\
\hline & & CONT2 & 0.880 & & \\
\hline & & CONT3 & 0.857 & & \\
\hline & & CONT4 & 0.821 & & \\
\hline & \multirow{5}{*}{ Conveniencia } & CONV1 & 0.633 & \multirow{5}{*}{0.880} & \multirow{5}{*}{0.597} \\
\hline & & CONV2 & 0.799 & & \\
\hline & & CONV3 & 0.795 & & \\
\hline & & CONV4 & 0.814 & & \\
\hline & & CONV5 & 0.807 & & \\
\hline & \multirow{4}{*}{ Credibilidad } & CRED1 & 0.849 & \multirow{4}{*}{0.901} & \multirow{4}{*}{0.695} \\
\hline & & CRED2 & 0.842 & & \\
\hline & & CRED3 & 0.846 & & \\
\hline & & CRED4 & 0.798 & & \\
\hline & \multirow{4}{*}{ Personalización } & PERS1 & 0.739 & \multirow{4}{*}{0.898} & \multirow{4}{*}{0.689} \\
\hline & & PERS2 & 0.875 & & \\
\hline & & PERS3 & 0.850 & & \\
\hline & & PERS4 & 0.850 & & \\
\hline \multirow{6}{*}{ Confianza } & & CONF1 & 0.856 & \multirow{6}{*}{0.932} & \multirow{6}{*}{0.697} \\
\hline & & CONF2 & 0.864 & & \\
\hline & & CONF3 & 0.869 & & \\
\hline & & CONF4 & 0.873 & & \\
\hline & & CONF5 & 0.709 & & \\
\hline & & CONF6 & 0.824 & & \\
\hline \multirow{5}{*}{ Lealtad } & & LEAL1 & 0.748 & \multirow{5}{*}{0.920} & \multirow{5}{*}{0.698} \\
\hline & & LEAL2 & 0.860 & & \\
\hline & & LEAL3 & 0.836 & & \\
\hline & & LEAL4 & 0.872 & & \\
\hline & & LEAL5 & 0.856 & & \\
\hline
\end{tabular}

Nota: La dimensión competencia fue eliminada debido a sus bajas cargas externas.

En el caso de la validez discriminante, misma que se muestra en la Tabla 4, se utilizó el criterio propuesto por Fornell y Larcker (1981), el valor de AVE evidenció la inter-correlación del constructo con otros en el modelo de investigación. Todos los valores fueron mayores que la correlación de cada uno de los constructos (Chin, 2010). Los resultados muestran, por lo tanto, que el modelo de medición fue satisfactorio y proporcionó evidencia suficiente en términos de confiabilidad, validez convergente y validez discriminante. El coeficiente de determinación $\left(\mathrm{R}^{2}\right)$ para confianza fue de 0.615 y para lealtad de 0.603 (para muestra completa), lo que explica en todos los casos más del $60 \%$ del constructo, respectivamente. Todos estos valores de $\mathrm{R}^{2}$ indican un modelo sustancial. 
Tabla 3. Validez Discriminante de los constructos-Cargas Cruzadas
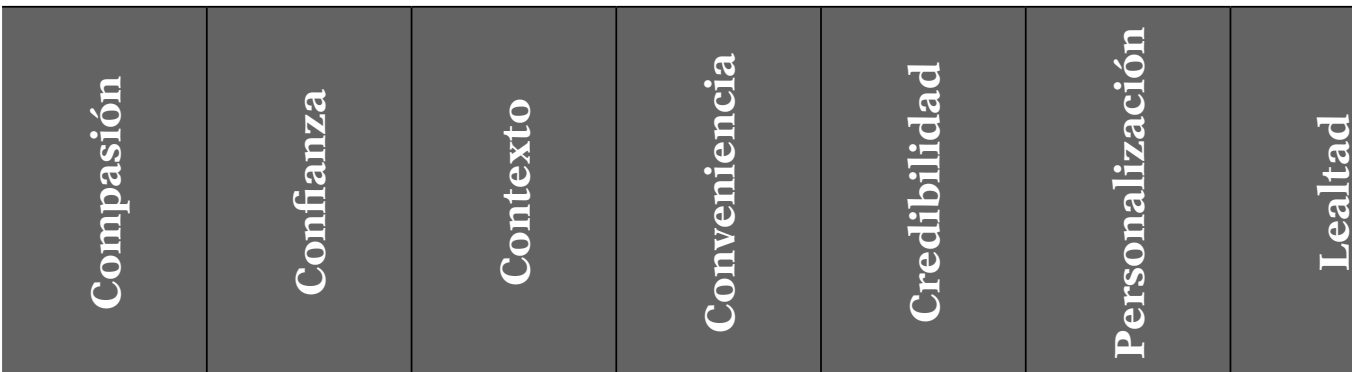

\begin{tabular}{|c|c|c|c|c|c|c|c|}
\hline Compa1 & 0,757 & 0,496 & 0,664 & 0,562 & 0,525 & 0,494 & 0,532 \\
\hline Compa1 & 0,757 & 0,496 & 0,664 & 0,562 & 0,525 & 0,494 & 0,532 \\
\hline Compa2 & 0,835 & 0,579 & 0,554 & 0,554 & 0,657 & 0,674 & 0,677 \\
\hline Compa2 & 0,835 & 0,579 & 0,554 & 0,554 & 0,657 & 0,674 & 0,677 \\
\hline Compa3 & 0,878 & 0,592 & 0,662 & 0,598 & 0,647 & 0,665 & 0,664 \\
\hline Compa3 & 0,878 & 0,592 & 0,662 & 0,598 & 0,647 & 0,665 & 0,664 \\
\hline Conf1 & 0,567 & 0,856 & 0,580 & 0,558 & 0,567 & 0,605 & 0,655 \\
\hline Conf2 & 0,607 & 0,864 & 0,606 & 0,585 & 0,587 & 0,628 & 0,670 \\
\hline Conf3 & 0,555 & 0,869 & 0,552 & 0,558 & 0,581 & 0,609 & 0,654 \\
\hline Conf4 & 0,594 & 0,873 & 0,594 & 0,593 & 0,609 & 0,652 & 0,680 \\
\hline Conf5 & 0,424 & 0,709 & 0,392 & 0,414 & 0,498 & 0,562 & 0,554 \\
\hline Conf6 & 0,613 & 0,824 & 0,607 & 0,586 & 0,561 & 0,610 & 0,668 \\
\hline Cont1 & 0,678 & 0,591 & 0,859 & 0,613 & 0,562 & 0,599 & 0,621 \\
\hline Cont1 & 0,678 & 0,591 & 0,859 & 0,613 & 0,562 & 0,599 & 0,621 \\
\hline Cont2 & 0,672 & 0,591 & 0,880 & 0,593 & 0,559 & 0,594 & 0,627 \\
\hline Cont2 & 0,672 & 0,591 & 0,880 & 0,593 & 0,559 & 0,594 & 0,627 \\
\hline Cont3 & 0,644 & 0,558 & 0,857 & 0,566 & 0,547 & 0,609 & 0,636 \\
\hline Cont3 & 0,644 & 0,558 & 0,857 & 0,566 & 0,547 & 0,609 & 0,636 \\
\hline Cont4 & 0,594 & 0,551 & 0,821 & 0,537 & 0,504 & 0,562 & 0,593 \\
\hline Cont4 & 0,594 & 0,551 & 0,821 & 0,537 & 0,504 & 0,562 & 0,593 \\
\hline Conv1 & 0,420 & 0,358 & 0,494 & 0,633 & 0,327 & 0,318 & 0,347 \\
\hline Conv1 & 0,420 & 0,358 & 0,494 & 0,633 & 0,327 & 0,318 & 0,347 \\
\hline Conv2 & 0,553 & 0,547 & 0,538 & 0,799 & 0,527 & 0,542 & 0,577 \\
\hline Conv2 & 0,553 & 0,547 & 0,538 & 0,799 & 0,527 & 0,542 & 0,577 \\
\hline Conv4 & 0,552 & 0,535 & 0,486 & 0,795 & 0,598 & 0,570 & 0,617 \\
\hline Conv4 & 0,552 & 0,535 & 0,486 & 0,795 & 0,598 & 0,570 & 0,617 \\
\hline Conv5 & 0,576 & 0,552 & 0,550 & 0,814 & 0,548 & 0,526 & 0,571 \\
\hline Conv5 & 0,576 & 0,552 & 0,550 & 0,814 & 0,548 & 0,526 & 0,571 \\
\hline Conv6 & 0,557 & 0,537 & 0,550 & 0,807 & 0,614 & 0,575 & 0,598 \\
\hline Conv6 & 0,557 & 0,537 & 0,550 & 0,807 & 0,614 & 0,575 & 0,598 \\
\hline Cred1 & 0,583 & 0,535 & 0,476 & 0,575 & 0,849 & 0,620 & 0,617 \\
\hline Cred1 & 0,583 & 0,535 & 0,476 & 0,575 & 0,849 & 0,620 & 0,617 \\
\hline Cred2 & 0,632 & 0,572 & 0,550 & 0,601 & 0,842 & 0,628 & 0,627 \\
\hline Cred2 & 0,632 & 0,572 & 0,550 & 0,601 & 0,842 & 0,628 & 0,627 \\
\hline
\end{tabular}



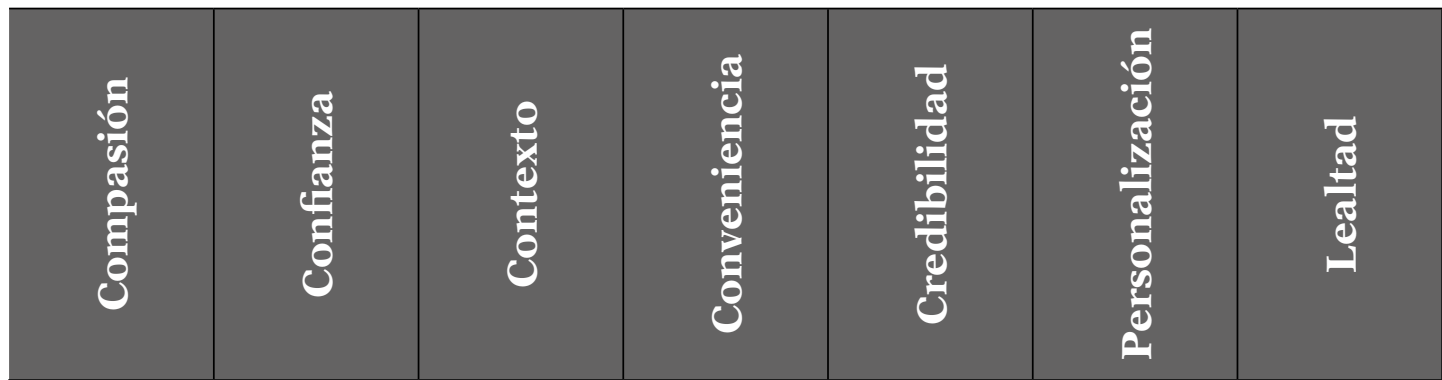

\begin{tabular}{|l|l|l|l|l|l|l|l|}
\cline { 2 - 8 } Cred3 & 0,644 & 0,595 & 0,546 & 0,573 & 0,846 & 0,665 & 0,646 \\
\hline Cred3 & 0,644 & 0,595 & 0,546 & 0,573 & 0,846 & 0,665 & 0,646 \\
\hline Cred4 & 0,613 & 0,567 & 0,548 & 0,543 & 0,798 & 0,620 & 0,616 \\
\hline Cred4 & 0,613 & 0,567 & 0,548 & 0,543 & 0,798 & 0,620 & 0,616 \\
\hline Leal1 & 0,602 & 0,590 & 0,541 & 0,521 & 0,581 & 0,651 & 0,748 \\
\hline Leal2 & 0,649 & 0,681 & 0,659 & 0,633 & 0,647 & 0,710 & 0,860 \\
\hline Leal3 & 0,585 & 0,628 & 0,528 & 0,546 & 0,622 & 0,700 & 0,836 \\
\hline Leal4 & 0,674 & 0,683 & 0,661 & 0,642 & 0,641 & 0,676 & 0,872 \\
\hline Leal5 & 0,660 & 0,659 & 0,628 & 0,624 & 0,646 & 0,673 & 0,856 \\
\hline Pers1 & 0,522 & 0,550 & 0,577 & 0,499 & 0,529 & 0,739 & 0,553 \\
\hline Pers1 & 0,522 & 0,550 & 0,577 & 0,499 & 0,529 & 0,739 & 0,553 \\
\hline Pers2 & 0,685 & 0,640 & 0,620 & 0,594 & 0,684 & 0,875 & 0,716 \\
\hline Pers2 & 0,685 & 0,640 & 0,620 & 0,594 & 0,684 & 0,875 & 0,716 \\
\hline Pers3 & 0,614 & 0,586 & 0,512 & 0,527 & 0,624 & 0,850 & 0,675 \\
\hline Pers3 & 0,614 & 0,586 & 0,512 & 0,527 & 0,624 & 0,850 & 0,675 \\
\hline Pers4 & 0,642 & 0,649 & 0,588 & 0,584 & 0,675 & 0,850 & 0,753 \\
\hline Pers4 & 0,642 & 0,649 & 0,588 & 0,584 & 0,675 & 0,850 & 0,753 \\
\hline
\end{tabular}

\section{Tabla 4. Validez Discriminante de los Constructos. Criterio de Fornell y Larcker}

\begin{tabular}{|c|c|c|c|c|c|c|c|}
\hline & 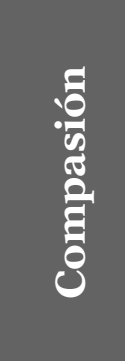 & 趈 & 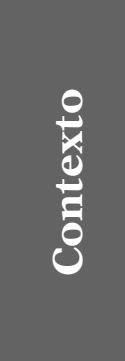 & $\begin{array}{l}\frac{9}{0} \\
\frac{0}{0} \\
\cdot \frac{0}{0} \\
0 \\
0\end{array}$ & 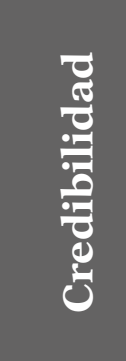 & 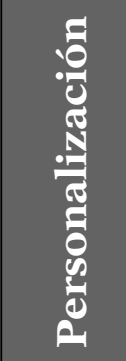 & $\frac{\mathscr{E}}{\mathscr{I}}$ \\
\hline Compasión & 0,825 & & & & & & \\
\hline Confianza & 0,675 & 0,835 & & & & & \\
\hline Contexto & 0,758 & 0,670 & 0,855 & & & & \\
\hline Conveniencia & 0,692 & 0,662 & 0,676 & 0,772 & & & \\
\hline Credibilidad & 0,741 & 0,681 & 0,636 & 0,688 & 0,834 & & \\
\hline Personalización & 0,745 & 0,732 & 0,692 & 0,666 & 0,760 & 0,830 & \\
\hline Lealtad & 0,760 & 0,777 & 0,724 & 0,712 & 0,752 & 0,816 & 0,835 \\
\hline
\end{tabular}

Nota: Los valores diagonales representan la raíz cuadrada de la varianza promedio extraída (AVE) mientras que las otras entradas representan las correlaciones. 


\subsection{Evaluación del modelo estructural}

En la Tabla 5 se presentan los resultados de las pruebas de hipótesis. La regla general para la prueba de hipótesis de una cola es que el valor de t debe exceder 1.965 $(\mathrm{p}<0.05)$. Se pudo evidenciar que la experiencia del cliente tiene un efecto positivamente significativo en la confianza (H1) y a su vez esta en la lealtad (H2), tal como se muestra en la Figura 1. Además, todas las dimensiones que son parte de la experiencia del cliente y fueron evaluadas, muestran una robustez significativa del constructo mencionado.

Tabla 5. Resultados de la evaluación estructural

\begin{tabular}{|l|l|l|l|l|}
\hline Hipótesis & \multicolumn{1}{|c|}{ Path } & Beta & Estadísticos t & p Valores \\
\hline H1 & Experiencia del cliente -> Confianza & 0,784 & 53,623 & 0,000 \\
\hline H2 & Confianza -> lealtad & 0,777 & 47,777 & 0,000 \\
\hline
\end{tabular}

Es necesario mencionar que como un criterio predictivo adicional al $\mathrm{R}^{2}$, autores como Leguina (2015) recomiendan el análisis de $\mathrm{Q}^{2}$, con el objetivo de valorar la relevancia predictiva del modelo estructural. Al respecto, Chin (1998) menciona que la relevancia predictiva de los constructos debe ser positiva y con valores mayores a cero; en donde, los valores de 0.02 se pueden considerar como valores pequeños, valores de 0.15 como valores medios y valores 0.35 como valores grandes para considerar validez predictiva del modelo. Geisser (1974) y Stone (1974) recomiendan evaluar la prueba de Stone-Geisser como un criterio de $\mathrm{Q}^{2}$. Esta investigación utilizó el procedimiento de blindfolding en PLS. Los constructos endógenos tuvieron una predicción fuerte, debido a que $\mathrm{Q}^{2}$ tuvo un valor 0.42 para el constructo confianza y un valor de 0.42 para lealtad. Finalmente, en la Tabla 6 se detallan los efectos indirectos entre experiencia y lealtad.

Tabla 6. Efectos indirectos experiencia-lealtad

\begin{tabular}{|l|l|l|l|l|l|}
\hline \multicolumn{1}{|c|}{} & $\begin{array}{c}\text { Muestra } \\
\text { original } \\
(\mathbf{O})\end{array}$ & $\begin{array}{c}\text { Media } \\
\text { de la } \\
\text { muestra } \\
\mathbf{( M )}\end{array}$ & $\begin{array}{c}\text { Desviación } \\
\text { estándar } \\
\text { (STDEV) }\end{array}$ & $\begin{array}{c}\text { Estadísticos t } \\
\text { (| O/STDEV|) }\end{array}$ & $\begin{array}{c}\text { P } \\
\text { Valores }\end{array}$ \\
\hline $\begin{array}{l}\text { Experiencia del cliente } \\
\text {-> lealtad }\end{array}$ & 0,609 & 0,610 & 0,022 & 27,070 & 0,000 \\
\hline
\end{tabular}




\section{Figura 1. Modelo estructural propuesto}

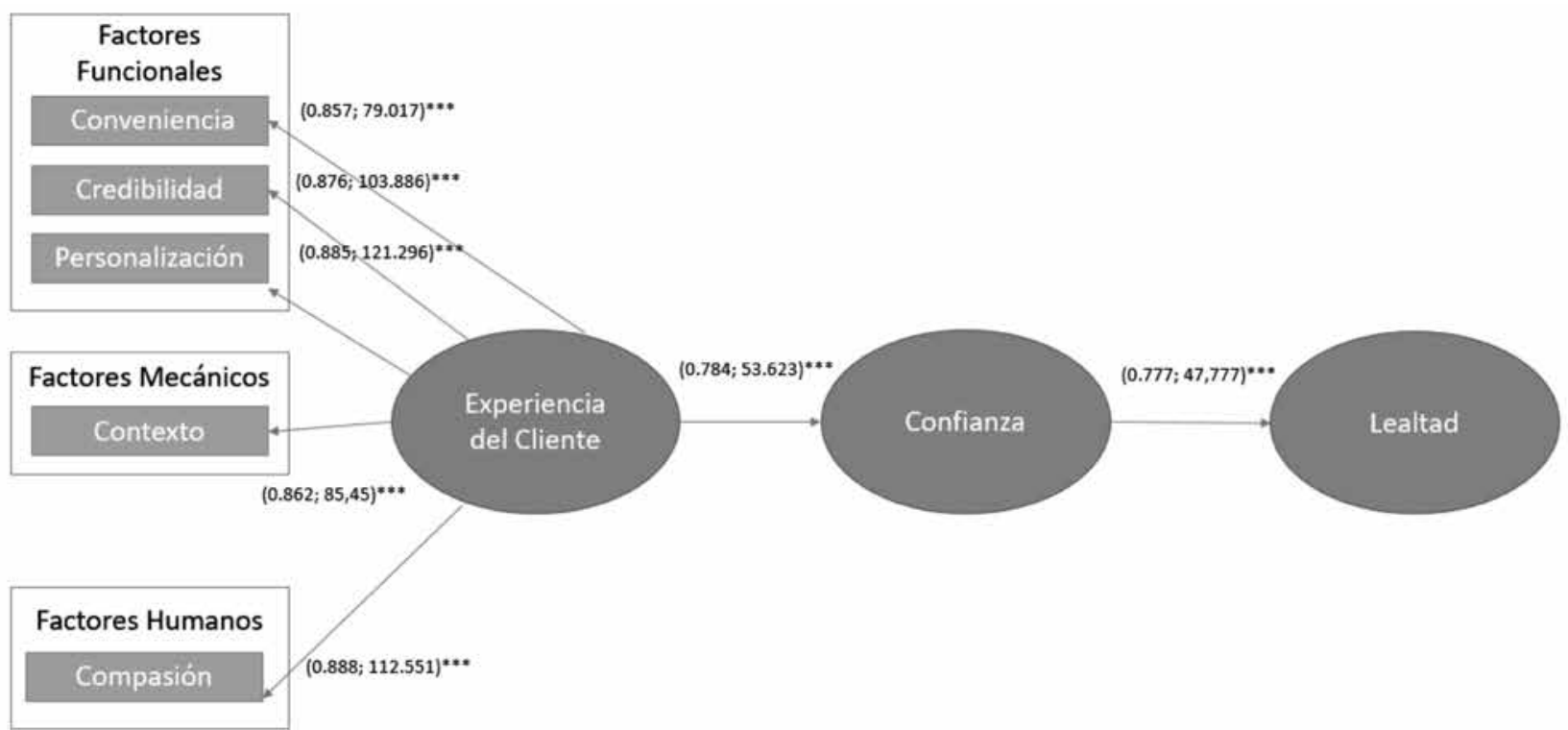

\section{Conclusiones y discusión}

Se ha investigado mucho con respecto a la experiencia del cliente, la confianza y la lealtad; Micu et al. (2019) indican que la experiencia del cliente en línea influye directamente en la confianza mientras que Omoregie et al. (2019) y Gillani y Awan (2014) coinciden en que la relación entre confianza y lealtad resulta significativa, sin embargo, no se han explorado los constructos que actúan como mediadores entre la experiencia del cliente y la lealtad tal como sugieren Kamath et al. (2019). En este sentido, este trabajo intentó cubrir la brecha descrita anteriormente.

$\mathrm{Al}$ momento de evaluar la experiencia del cliente, se encontró que las dimensiones conveniencia, credibilidad, personalización, contexto y compasión, fortalecen el constructo, el efecto de todas ellas en la experiencia del cliente es sumamente fuerte y positivo. Como indica Wasan (2018), si bien el juicio lo realizan los clientes sobre su experiencia general del servicio, esta se forma considerando varios factores de tipo funcional, humano y mecánico; el primero de ellos, se mide particularmente en términos de su confiabilidad y competencia; esto resulta interesante dado que esta investigación confirmó que la dimensión de competencia no era representativa en la experiencia del cliente.

Los resultados de la evaluación estructural que se informan en la Tabla 5 (resultados para la relación directa), demuestran que la experiencia del cliente tuvo un efecto significativamente positivo en la confianza. A partir de lo propuesto por Slåtten et al. (2011) quienes indican que las claves al momento de construir una mejor experiencia para el usuario, son provocar emociones y reacciones experienciales del consumidor, generando un vínculo estrecho entre ellos, se puede considerar que como indican Ali et al. (2018) las experiencias placenteras pueden ser determinantes de la lealtad del cliente, por lo tanto, generadoras de confianza también.

En el caso de la confianza, esta tuvo un efecto significativamente positivo en la lealtad de los millennials específicamente en el sector bancario. Los resultados son consistentes con las conclusiones de Gillani y Awan (2014) quienes confirman una relación significativa entre la confianza y la fidelidad del cliente, por lo que se demuestra que la confianza representa una variable que antecede a la lealtad. Los rasgos y comportamientos de los millennials al ser conocedores de la tecnología y expertos en usar 
Internet para la búsqueda y compra de productos, evidencian una relación entre las variables que son parte del modelo propuesto aplicado en el sector bancario.

En conclusión, el estudio destaca en primera instancia la fiabilidad del constructo experiencia del cliente porque su estructura - que contempla factores funcionales, mecánicos y humanos - resulta consistente. El efecto de la experiencia del cliente sobre la confianza es muy fuerte por lo que se confirma que la creación de recuerdos memorables a lo largo de todo el proceso de servicio, colaboran a la generación de confianza por parte de los millennials de la ciudad de Cuenca. El efecto de la confianza en la lealtad, es fuerte y positivo, por lo que los usuarios de la banca una vez que logran experimentar confianza en el servicio, empiezan a construir una relación de lealtad para con la marca.

Cada vez más, los bancos entienden la relación entre los constructos que forman parte del modelo y desarrollan esfuerzos para mejorar la experiencia del usuario tanto en el formato físico como el virtual. Esta investigación aporta significativamente al sector bancario, ya que proporciona información vital para la creación de ventajas competitivas en el sector y para el desarrollo de estrategias que incrementen la fidelidad de los usuarios actuales.

\section{Referencias}

Ali, F., Kim, W. G., Li, J., \& Jeon, H.M. (2018). Make it delightful: Customers' experience, satisfaction and loyalty in Malaysian theme parks. Journal of Destination Marketing $\mathcal{E}$ Management, 7, 1-11. https://doi.org/10.1016/j.jdmm.2016.05.003

Agarwal, R. (2020). Transformación digital: un camino al valor económico y social. Revista CEA, Ciencias Económicas y Administrativas, 6(12), 9-12. https://doi.org/10.22430/24223182.1700

Arora, S., \& Sandhu, S. (2018). Usage based upon reasons: the case of electronic banking services in India. International Journal of Bank Marketing, 36(4), 680-700. https://doi.org/10.1108/IJBM-032017-0060

Bhattacherjee, A. (2002). Individual trust in online firm: scale development initial test. Journal of Management Information Systems 19 (1): 211-241. https://doi.org/10.1080/07421222.2002.11045715

Bagozzi, R.P., Yi, Y., \& Phillips, L.W. (1991). Assessing Construct Validity in Organizational Research. Administrative Science Quarterly, 36(3), 421. https://doi.org/10.2307/2393203

Bashir, I., \& Madhavaiah, C. (2015). Consumer attitude and behavioural intention towards Internet banking adoption in India. Journal of Indian Business Researc, 7(1), 67-102. https://doi. org/10.1108/jibr-02-2014-0013

Brakus, J.J., Schmitt, B.H., \& Zarantonello, L. (2009). Brand Experience: What is It? How is it Measured? Does it Affect Loyalty? Journal of Marketing, 73(3), 52-68. https://doi.org/10.1509/ jmkg.73.3.052

Buttle, F. (2008). Customer relationship management: concepts and technologies. https://doi. org/10.4324/9780080949611

Chin, W. (1998). The partial least square approach to structural equation modelling. En G. Marcoulides (Ed.), Modern Methods for Business Research (pp. 295-369). Lawrence Erlbaum.

Chin, W.W. (2010). How to write up and report PLS analyses. In Esposito, V.V., Chin, W., Henseler, J., Wang, H. (Eds.), Handbook of Partial Least Squares: Concepts, Methods and Application (pp. 645689). Springer.

De Keyser, A. (2015). Understanding and managing the customer experience. PhD Series Ghent University. Faculty of Economics and Business Administration. Ghent University. Faculty of Economics and Business Administration, Ghent, Belgium.

Dabholkar, P., \& Overby, J. (2005). Linking process and outcome to service quality and customer satisfaction evaluations: an investigation of real estate agent service. International Journal of Service Industry Management, , 16(1), 10-27. https://doi.org/10.1108/09564230510587131 
Doney, P., \& Cannon, J. (1997). An examination of the nature of trust in buyer-seller. Journal of Marketing, 35-51. https://doi.org/10.1177/002224299706100203

Drigă, I., \& Isac, C. (2014). E-banking services-features, challenges and benefits. Annals of the University of Petroşani Economics, 14(1), 41-50. https://bit.ly/3ij5PIA

Fornell, C., Larcker, D.F. (1981). Evaluating structural equation models with unobservable variables and measurement error. Journal of Marketing Research, 18 (1), 39-50. https://doi. org/10.2307/3151312

Geisser, S. (1974). A predictive approach to the random effects model. Biometrika, 61, 101-107. doi: https://doi.org/10.1093/biomet/61.1.101

Gillani, S.U.A., \& Awan, A.G. (2014). Customer Loyalty in Financial Sector: A case study of Commercial Banks in Southern Punjab. International Journal of Accounting and Financial Reporting, 1(1), 587. https://doi.org/10.5296/ijafr.v4i2.6870

INEC (2019). Instituto Nacional de Estadisticas y Censos. https://bit.ly/2TD7kad

Jarvenpaa, S., Tractinsky, N., \& Vitale, M. (2000). Consumer trust in an Internet store. Information Technology and Management, 1(1/2), 45-71. https://doi.org/10.1023/A:1019104520776

Kamath, P.R., Pai, Y.P., \& Prabhu, N.K.P. (2019). Building customer loyalty in retail banking: a serial-mediation approach. International Journal of Bank Marketing. https://doi.org/10.1108/IJBM01-2019-0034

Kaur, S., \& Arora, S. (2020). Role of perceived risk in online banking and its impact on behavioral intention: trust as a moderator. Journal of Asia Business Studies. doi: https://doi.org/10.1108/ JABS-08-2019-0252

Kavitha, S., \& Haritha, P. (2018). A Study on Customer Experience and its Relationship with Repurchase Intention among Telecom Subscribers in Coimbatore District. International Journal of Management Studies, V3(3), 83. https://doi.org/10.18843/ijms/v5i3(3)/11

Lancaster, L., \& Stillman, D. (2002), When Generations Collide. Harper Collins Publishing.

Leguina, A. (2015) A primer on partial least squares structural equation modeling (PLSSEM). International Journal of Research $\mathcal{E}$ Method in Education, 38, 2, 220-221, https://doi.org/10.1080/1743727X.2015.1005806

Leninkumar, V. (2017). The Relationship between Customer Satisfaction and Customer Trust on Customer Loyalty. International Journal of Academic Research in Business and Social Sciences, 7(4). https://doi.org/10.6007/ijarbss/v7-i4/2821

Loureiro, S., \& Miranda, F. J. \& Breazeale, M. (2014). Who Needs Delight? The Greater Impact of Value, Trust and Satisfaction in Utilitarian, Frequent-Use Retail. Journal of Service Management, 25. https://doi.org/10.1108/josm-06-2012-0106

Mahecha-Freyle, O.J., López-Juvinao, D.D., \& Socarras-Bertiz, C.A. (2019). Estrategias competitivas de marketing financiero en el sector bancario: Percepción del cliente. Revista Venezolana de Gerencia, 24(88), 1166-1178. https://doi.org/10.37960/revista.v24i88.30171

Mbama, C.I., Ezepue, P., Alboul, L., \& Beer, M. (2018). Digital banking, customer experience and financial performance: UK bank managers' perceptions. Journal of Research in Interactive Marketing, 12(4), 432-451. https://doi.org/10.1108/JRIM-01-2018-0026

Micu, A. E., Bouzaabia, O., Bouzaabia, R., Micu, A., \& Capatina, A. (2019). Online customer experience in e-retailing: implications for web entrepreneurship. International Entrepreneurship and Management Journal, 15(2), 651-675. https://doi.org/10.1007/s11365-019-00564-X

Moliner-Tena, M.A., Monferrer-Tirado, D., \& Estrada-Guillén, M. (2019). Customer engagement, non-transactional behaviors and experience in services: A study in the bank sector. International Journal of Bank Marketing, 37(3), 730-754. https://doi.org/10.1108/IJBM-04-2018-0107

Nowak, L., Thach, L., \& Olsen, J. E. (2006). Wowing the millennials: Creating brand equity in the wine industry. Journal of Product and Brand Management, 15(5), 316-323. https://doi. org/10.1108/10610420610685712

Omoregie, O.K., Addae, J.A., Coffie, S., Ampong, G.O. A., \& Ofori, K.S. (2019). Factors influencing consumer loyalty: evidence from the Ghanaian retail banking industry. International Journal of Bank Marketing, 37(3), 798-820. https://doi.org/10.1108/IJBM-04-2018-0099 
Pavlou, P.A. (2003). Consumer Intentions to Adopt Electronic Commerce-Incorporating Trust and Risk in the Technology Acceptance Model. International Journal of Electronic Commerce,7(3), 101123. https://doi.org/10.1080/10864415.2003.11044275

Pikkarainen, K., Pikkarainen, T., Karjaluoto, H., \& Pahnila, S. (2006). The measurement of enduser computing satisfaction of online banking services: empirical evidence from Finland. International Journal of Bank Marketing, 24(3), 158-172. https://doi.org/10.1108/02652320610659012

Roy, M., Dewit, O., \& Aubert, B. (2001). The impact of interface usability on trust in web retailers. Internet Research: Electronic Networking Applications and Policy, 11(5), 388-398. https://doi. org/10.1108/10662240110410165

Sáenz, K., \& Tamez, G. (2014). Modelo de ecuaciones estructurales por el método de mínimos cuadrados parciales. En Métodos y técnicas cualitativas y cuantitativas aplicables a la investigación en Ciencias Sociales. Tirant Humanidades.

Safeena, R., Date, H., Hundewale, N., \& Kammani, A. (2013). Combination of TAM and TPB in electronic banking adoption. International Journal of Computer Theory and Engineering, 5(1), 146. https://doi.org/10.7763/ijcte.2013.v5.665

San Martín, S., \& Camarero, C. (2010): Los determinantes de la confianza del comprador online. Comparación con el caso subasta. Cuadernos de Gestión, 10, número especial, 43-61. https://doi. org/10.5295/cdg.100187ss

Sarin, S., Sego, T., \& Chanvarasuth, N. (2003). Strategic use of bundling for reducing consumers' perceived risk associated with the purchase of new high-tech products. Journal of Marketing Theory and Practice, 11(3), 71-83. https://doi.org/10.1080/10696679.2003.11658502

Shamsuddoha, M., \& Alamgir, M. (2003). Loyalty and satisfaction construct in retail banking - an empirical study on bank customers. The Chittagong University Journal of Business Administration, 19. https://ssrn.com/abstract=1295427

Slåtten, T., Krogh, C., \& Connolley, S. (2011). Make it memorable: customer experiences in winter amusement parks. International Journal of Culture, Tourism and Hospitality Research, 5(1), 80-91. https://doi.org/10.1108/17506181111111780

Srivastava, M., \& Kaul, D. (2016). Exploring the link between customer experience-loyalty-consumer spend. Journal of Retailing and Consumer Services, 31, 277-286. https://doi.org/10.1016/j.jretconser.2016.04.009

Stone, M. (1974). Cross-validatory choice and assessment of statistical predictions. Journal of the Royal Statistical Society, 36, 111-147. https://doi.org/10.1111/j.2517-6161.1976.tb01573.x

Suh, B., \& Han, I. (2002). Effect of trust on customer acceptance of Internet banking. Electronic Commerce Research and Applications, 1 (3/4), 247-263. https://doi.org/10.1016/s1567-4223(02)00017-0

Torres, E., Manzur, E., Olavarrieta, S., \& Barra, C. (2009). Análisis de la relación confianza-compromiso en la banca en internet. Revista Venezolana de Gerencia, 14(47), 371-392. https://doi. org/10.31876/revista.v14i47.10539

Verhoef, P.C., Lemon, K.N., Parasuraman, A., Roggeveen, A., Tsiros, M., \& Schlesinger, L.A. (2009). Customer experience creation: determinants, dynamics and management strategies. Journal of Retailing, 85(1), 31-41. https://doi.org/10.1016/j.jretai.2008.11.001

Wasan, P. (2018). Predicting customer experience and discretionary behaviors of bank customers in India. International Journal of Bank Marketing, 36(4), 701-725. https://doi.org/10.1108/ijbm-062017-0121 